\title{
GAS NATURAL LICUADO PARA EL TRANSPORTE MARÍTIMO DE CORTA DISTANCIA: ESTUDIO MEDIANTE REDES BAYESIANAS DE LAS VARIABLES ECONÓMICAS
}

\section{LIQUEFIED NATURAL GAS FOR SHORT SEA SHIPPING: STUDY OF ECONOMIC VARIABLES USING BAYESIAN NETWORKS}

\author{
Beatriz Molina Serrano ${ }^{1,}$, Nicoleta González-Cancelas², Francisco Soler-Flores ${ }^{3}$
}

\section{RESUMEN}

En los últimos tiempos las implicaciones de la reglamentación medioambiental en el sector transporte han ido en aumento, la Comunidad Europea se ha marcado como objetivo llevar a cabo una autentica estrategia para racionalizar la política de transportes, así ha ocurrido también en el transporte marítimo, con especial incidencia ante la posibilidad de ampliación de las zonas de navegación ECAs (Emission Control Area) en todo el mundo y las nuevas normativas de reducción de contenido de azufre de la Unión Europea. Este marco de trabajo en el que opera el sector portuario está favoreciendo la consolidación del GNL (gas natural licuado) como un combustible alternativo para el transporte de corta distancia o short sea shipping.

El estudio que se presenta pretende analizar de la implicación de variables económicas incidentes en la elección del gas natural licuado como combustible en el transporte marítimo de corta distancia mediante el uso de redes bayesianas. La metodología empleada ha hecho necesaria la construcción de una red bayesiana, empleando un total de 35 indicadores, los cuales se han clasificado en 5 categorías distintas. Analizando dicha red se ha obtenidos que las variables "Capacidad de las terminales de regasificación de GNL en construcción" y "Distribución modal de transporte de carga por aguas continentales" son los dos nodos raíz de la red, de forma que ambas categorías resultan significativas a la hora de decidir la implementación del gas natural como combustible. Por el contrario, las categorías de medio ambiente y energía y de población y condición social son las más dependientes, al ser las categorías más alejadas de los nodos raíz de la red.

Palabras claves: Redes bayesianas, gas natural licuado, GLN, transporte marítimo de corta distancia, Área de Control de Emisiones, Áreas de Control de Emisiones de Sulfuros.

\footnotetext{
${ }^{1}$ Departamento de Ingenieria Civil. Transportes. Escuela Técnica Superior de Ingenieros de Caminos, Canales y Puertos. Universidad Politécnica de Madrid. Madrid, España. orcid.org/0000-0002-78329573

${ }^{2}$ Departamento de Ingenieria Civil. Transportes. Escuela Técnica Superior de Ingenieros de Caminos, Canales y Puertos. Universidad Politécnica de Madrid. Madrid, España. orcid.org/0000-0001-71671563

${ }^{3}$ Facultad de Educación. Universidad Internacional de La Rioja. La Rioja, España. orcid.org/0000-0002-1636-834X
} 


\section{ABSTRACT}

In recent times the implications of environmental regulation in the transport sector have been increasing. The European Community has set itself the goal of carrying out an authentic strategy to rationalize transport policy, this has also happened in maritime transport, with special impact due to the possibility of expanding the navigation zones ECAs (Emission Control Area) around the world and the new regulations for reduction of sulfur content of the European Union. This framework in which the port sector operates is favoring the consolidation of LNG (liquefied natural gas) as an alternative fuel for short sea shipping.

The present study aims to analyze the implication of incidental economic variables in the choice of liquefied natural gas as fuel in short sea shipping through the use of Bayesian networks. Used methodology has made it necessary to construct a Bayesian network using 35 indicators. They have been classified in 5 different categories. Result of network analysis show that variables called "Capacity of liquefied natural gas regasification terminals under construction" and "Modal distribution of inland water goods transportation" are the two root nodes. So, both of them are significant variables to make decision of liquefied natural gas implementation as fuel. However, environmental and energy category and population and social condition category are the most dependent ones because they are far away from root nodes in the network.

Keyword: Bayesian Networks, liquid natural gas, liquefied natural gas, short sea shipping, Emission Control Area, Sulphur Emission Control Areas.

\section{INTRODUCCIÓN}

El crecimiento del comercio internacional de los años 90 del siglo pasado y la entrada de nuevos países a la Unión Europea conlleva un aumento en la congestión de los principales corredores europeos, amenazando la competitividad económica, al introducir costes adicionales a la productividad de las empresas. Ante esto, y dados los elevados costes de las externalidades ocasionadas por el transporte terrestre (principalmente contaminación y accidentalidad), se plantea a nivel continental una estrategia que consiste en transportar las mercancías por vía marítima la mayor distancia posible, pues con dicho planteamiento se considera que se consiguen reducir los daños ambientales, la accidentalidad y la saturación de la infraestructura, al tiempo que se incrementa la eficiencia energética (Cánovas, 2015).

Al mismo tiempo, con el impulso del transporte marítimo de corta distancia se espera optimizar los modos de transporte, descongestionar las carreteras, reducir las emisiones de CO2 y SOx que afectan el medio ambiente y la salud humana, crear nuevas rutas entre puertos europeos o vecinos y ofrecer un transporte seguro y de calidad. Asimismo, se espera garantizar la conexión entre el mar, las vías navegables y el ferrocarril, a través de la intermodalidad, además de desarrollar las autopistas del mar (Comisión Europea, 2001).

De esta manera, el transporte marítimo se convierte en un complemento al transporte de mercancías por carretera, logrando incrementar el rendimiento y capacidad del sistema en general. En los proyectos RECORDIT (2003) y REALISE (2002 y 2005) se demuestra que el transporte intermodal reduce los costos internos y externos totales, lo que hace que este se 
convierta en una mejor alternativa económica cuando se compara con el transporte unimodal (Lloyd, 2003; Vassallo, 2004). Por esta razón, la Comisión Europea promueve el Short Sea Shipping (SSS) como una alternativa al transporte unimodal, puesto que complementa el transporte de carga y pasajeros por carretera, es más eficiente energéticamente, contamina menos y responde a la congestión de las infraestructuras viales europeas. Es posible definir el SSS como el transporte de mercancías y pasajeros entre los puertos del continente o entre la Unión Europea y puertos de otros países situados en su área de influencia en el Mar Báltico, Mediterráneo o Negro.

Sin embargo, el resultado no es el esperado, ya que esta estrategia aumenta la contaminación adyacente a las costas del continente, provocada por el combustible usado en las embarcaciones de clase feeder, el cual produce no solamente emisiones de gases de efecto invernadero tipo $\mathrm{CO} 2$, sino también altos niveles de emisión de partículas de óxido de azufre (Comisión Europea, 2001). Por este motivo se establece una política energética que propusiera soluciones sobre el abastecimiento, los efectos del crecimiento y el medio ambiente europeo (Comisión Europea, 2006), así como la creación de una industria marítima respetuosa con el medio ambiente, competitiva, innovadora y complementaria, surgiendo también la necesidad de buscar combustibles alternativos al comúnmente usado en el transporte marítimo de corta distancia en Europa, con el objetivo de reducir los niveles de contaminación en zonas costeras densamente pobladas y con presencia de alto tráfico de embarcaciones.

Psaraftis \& Kontovas (2010) afirman que existen diversas maneras de reducir los gases de efecto invernadero tales como tener motores de bajo consumo, encontrar una propulsión óptima, usar combustibles alternativos o depuradores en el escape, etc. Otras contribuciones interesantes se han realizado en torno al impacto medioambiental del SSS y las recomendaciones de políticas sobre el cambio climático, dentro de las que se encuentran los desarrollos de Corbett et al.(1999), Endresen (2008), Corbett \& Koehler (2003), Corbett et al., (2009), Devanney (2010), Devanney \& Beach (2010). No obstante, existe una ausencia de desarrollos sobre el impacto financiero de la regulación en el sector del transporte marítimo (Almazán et al., 2013).

Uno de los combustibles potenciales a usar en un futuro próximo es el Gas Natural Licuado (GNL) que cuenta con emisiones de CO2 ligeramente inferiores al tradicional y, principalmente, con ausencia de emisiones de Dióxido de Sulfuro SO2, así como de Óxido de Nitrógeno NOx (Brynolf et al., 2011). Aunque los beneficios son numerosos, el número de barcos que actualmente usan el GNL aún no es significativo.

Los estudios e investigaciones realizados con respecto al tipo de combustible usado en el transporte marítimo de corta distancia en Europa que podrían cumplir las exigencias medioambientales de contenido de sulfuros, se concentran básicamente en tres: el fuel oil pesado (HFO), gasóleo marino (MGO) y el gas natural licuado (GNL). El primero, es el más usado en la actualidad a pesar de tener un contenido de azufre de al menos el $1 \%$ de la masa, es decir, más del permitido en las Áreas de Control de Emisiones de Sulfuro. Aunque los dos siguientes cumplen con las regulaciones, el último resulta ser la mejor alternativa no solo por cumplir con las estrictas reglamentaciones para emisiones de dióxido de sulfuro SOx, sino también por satisfacer aquellas relacionadas con las de Óxidos de Nitrógeno NOx y CO2 (Brynolf et al., 2011).

Con respecto a las emisiones de gases de efecto invernadero, el GNL produce una cantidad ligeramente inferior a la generada por los combustibles tipo diésel y es solamente un $1 \%$ menos eficiente. No obstante, su implementación requiere una inversión significativa en 
barcos cargueros de GNL de mediana y pequeña escala para llevar el gas a las terminales intermedias o a los tanques de almacenamiento y su estricta regulación ambiental desafía las formas de gobernanza marítima tradicionales (Verbeek et al., 2011; Gullberg \& Gahnström, 2011; Gritsenko \& Yliskylä-Peuralahti, 2013; Acedo \& Almazán, 2015).

Dado que los estudios que han propuesto analizar el cambio de combustible en este tipo de embarcaciones, de acuerdo a nuestro conocimiento, son escasos, al igual que la literatura que relaciona el GNL con el transporte marítimo, el objetivo de la presente investigación fue elaborar una metodología basada en redes bayesianas para determinar la relación entre las variables que definen el uso del GNL en el SSS en Europa. Este tipo de redes representan, de manera compacta, una distribución de probabilidad multivariante. El uso de redes bayesianas para la representación gráfica de las relaciones entre variables trae consigo una gran cantidad de ventajas, que facilitan la comprensión de las relaciones causales a través del grafo (Puga et al., 2007). Algunas de estas son:

- Este tipo de redes permite representar de forma simultánea la dimensión cuantitativa (tablas de probabilidad) y cualitativa (grafo) de un problema

- Las redes bayesianas, pueden ser trabajadas con datos incompletos (Puga et al., 2007) (Heckerman, 2008)

- Es posible realizar una inferencia bidireccional (Rodríguez \& Dolado, 2007), (Pearl, 2011), (Puga et al., 2007)

- Es posible realizar un análisis de sensibilidad (Rodríguez \& Dolado, 2007), (Laskey, 2014)

- Las redes bayesianas pueden realizar predicciones con información incompleta, entre otras.

A partir de los resultados, será posible establecer las variables económicas más relevantes en la decisión de implementar el GNL como combustible en el tramo marítimo de las autopistas del mar y aquellas que no lo son.

Los modelos gráficos probabilísticos son gráficos en los que los nodos representan variables aleatorias, y los arcos (falta de) representan supuestos de independencia condicional. Por lo tanto, proporcionan una representación de distribuciones de probabilidad conjuntas. Estos modelos gráficos pueden ser de dos tipos:

- Modelos gráficos no dirigidos. Estos modelos tienen una definición simple de independencia, es decir, dos (conjuntos de) nodos A y B son condicionalmente independientes dado un tercer conjunto, $\mathrm{C}$, si todos los caminos entre los nodos en $\mathrm{A}$ y $\mathrm{B}$ están separados por un nodo en $\mathrm{C}$.

- Modelos gráficos dirigidos. Estos modelos también son conocidos como redes Bayesianas. Tienen una noción más complicada de la independencia, dado que tiene en cuenta la direccionalidad de los arcos.

Aunque los modelos dirigidos tienen una noción más complicada de independencia, su principal ventaja es que se puede considerar un arco de A a B como indicando que A "causa" $\mathrm{B}$, lo cual puede usarse como una guía para construir la estructura del gráfico. Además, este 
tipo de modelos permite codificar relaciones deterministas, siendo más fácil de ajustar a los datos.

Un estudio pormenorizado de la relación entre modelos gráficos dirigidos y no dirigidos es posible encontrarla en las investigaciones de (Shachter, 1988), (Pearl, 1982) y (Castillo et al., 1997).

Se puede decir que una Red Bayesiana es un conjunto de nodos y arcos donde cada nodo corresponde a una variable que representa una entidad del mundo real. Los arcos de unión de dichos nodos indican relaciones de influencia causal entre las variables (Flores et. al, 2013). A nivel técnico, una red bayesiana es un grafo dirigido acíclico en donde cada uno de sus nodos hace referencia a una variable aleatoria y las relaciones entre las variables se encuentran codificadas en la propia estructura del grafo de acuerdo al criterio de separación. A cada nodo de la red se le asocia una distribución de probabilidad condicionada a los padres de ese nodo, de tal forma que la distribución conjunta multiplica las distribuciones condicionadas asociadas a los nodos de la red; la ecuación (1) muestra una red con $n$ variables $X_{1}, X_{2}, \ldots . . X_{n}$.

$$
p\left(x_{1}, \ldots, x_{n}\right)=\prod_{i=1}^{n} p\left(x_{1} \mid x_{p a(i)}\right)
$$

Rodríguez y Dolado (2007) aseguran que la ingeniería del conocimiento en las Redes Bayesianas es el proceso de construcción, validación y uso de las redes Bayesianas. Este, se realiza por medio de la definición de la estructura o grafo y de los parámetros de la red, para lo cual es necesario seguir seis pasos: conocer el dominio a modelar, seleccionar las variables de interés, definir un tipo para cada variable, establecer la topología de la red, construir las tablas de probabilidad para cada nodo y, finalmente evaluar y verificar la red bayesiana. Así, para la construcción de la red es necesario definir tanto la estructura gráfica, como los parámetros, de igual forma que debe hacerse para la construcción de cualquier tipo de modelo. Sin embargo, al tratarse de un modelo dirigido, se debe especificar la distribución de probabilidad condicional en cada nodo.

Cuando el sistema contiene un número pequeño de variables y estados, la construcción de la red bayesiana puede realizarse de forma manual. No obstante, en la actualidad se ha vuelto necesario automatizar el proceso de construcción de la red porque la mayoría de las bases de datos contienen un elevado número de variables que dificultan la construcción manual (Rodríguez \& Dolado, 2007). Dicha automatización puede realizarse a través de los pasos de la técnica de minería de datos, que son herramientas que ha surgido para procesar grandes cantidades de información (Lizazo Torres et. al, 2011). En el estudio, el software empleado para la construcción de la red fue Elvira, al contar con un formato para la codificación de los modelos, un lector-intérprete para los modelos codificados, una interfaz para la construcción de redes, algoritmos para la toma de decisiones, entre otros.

\section{MATERIAL Y MÉTODOS}

La metodología empleada en la investigación se dividió en tres etapas (Figura 1). 

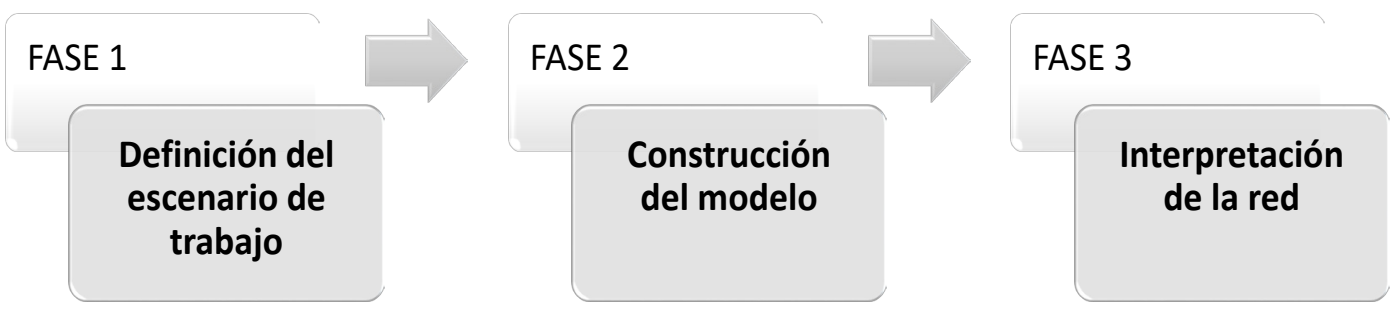

Figura 1. Representación esquemática de la metodología empleada

\section{Fase 1: Definición del escenario de trabajo}

El primer paso para la descripción del escenario de trabajo, tras realizar una revisión de la bibliografía existente, fue la delimitación del ámbito del estudio. La delimitación geográfica fueron los países europeos, habiendo considerado un total de 30 países. De estos 30 países, 28 correspondieron a los miembros de la Unión Europea, siendo los dos restantes Noruega e Islandia. La inclusión de estos países se debió a que además de ser países marítimos, tienen características comunes a los de la Unión Europea, así, por ejemplo, Noruega pertenece a la SECA del Mar del Norte.

Una vez establecido el ámbito geográfico, se procede a la determinación y obtención de las variables que participan en el estudio. En el caso considerado, las variables se seleccionaron de acuerdo a la relación directa o indirecta que tuviesen con el GNL y las autopistas del mar, a partir de un panel de expertos. Así, en total se incluyeron un total de 35 indicadores (Figura 2).

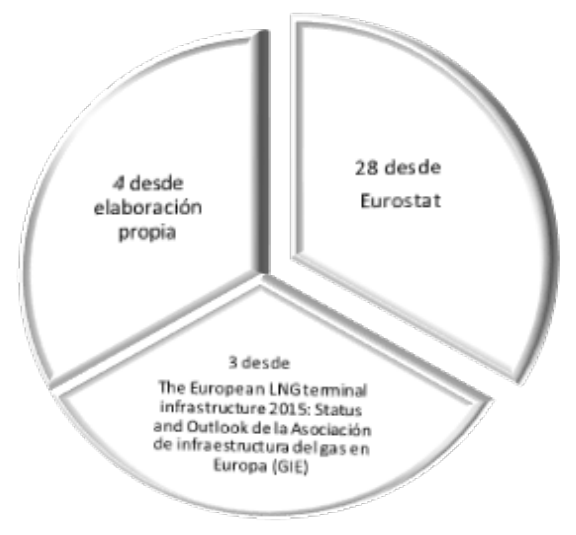

Figura 2. Distribución de los indicadores considerados en función de sus fuentes

El siguiente paso consistió en la clasificación de estas variables en grupos homogéneos en función de su naturaleza, con objeto de construir la base de datos a partir de la cual se generará el modelo. Así, los 35 indicadores se agruparon en los grupos siguientes (Figura 3). 


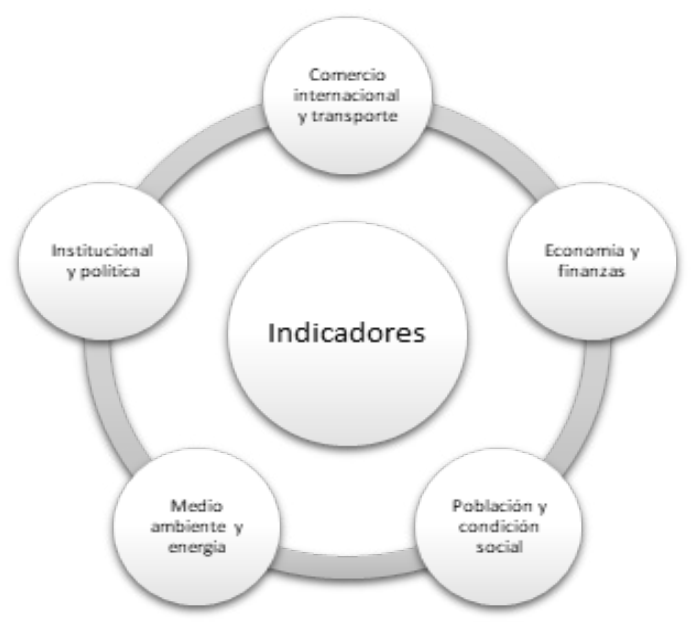

Figura 3. Clasificación de los indicadores considerados en función de su naturaleza

Por tanto, una vez seleccionadas las variables a través de un panel de expertos, y habiéndolas agrupado en categorías, se confeccionó una base de datos que da origen a la red bayesiana con variables susceptibles de relacionarse con el gas natural licuado en el sector transportes y, de una forma más específica, con el transporte marítimo de corta distancia en particular. El conjunto de variables consideradas que conforman la base de datos, clasificadas en función de las categorías definidas se presentan en la Tabla 1.

Tabla 1. Variables y categorías del proyecto

\begin{tabular}{|c|c|c|c|c|}
\hline Categoría & Indicador & Variable & $\begin{array}{l}\text { Unidad } \\
\text { medida }\end{array}$ & de \\
\hline \multirow{5}{*}{ 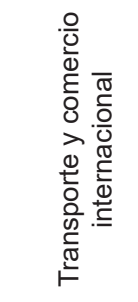 } & Transporte Marítimo & MTG & $\begin{array}{l}\text { Miles } \\
\text { toneladas }\end{array}$ & de \\
\hline & Distribución modal de transporte de carga por ferrocarril & MSFT RAIL & Porcentaje & \\
\hline & Distribución modal de transporte de carga por carretera & MSFT ROAD & Porcentaje & \\
\hline & $\begin{array}{l}\text { Distribución modal de transporte de carga por aguas } \\
\text { continentales }\end{array}$ & MSFT INW & Porcentaje & \\
\hline & TEUs en short sea shipping & SSS VTEU & 1000 TEU's & \\
\hline \multirow{8}{*}{ 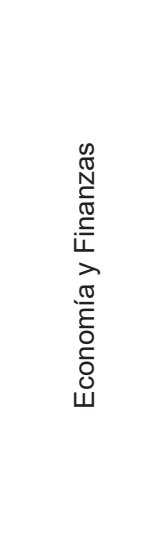 } & Producto interno bruto & GDP & $\begin{array}{l}\text { Millones } \\
\text { Euros }\end{array}$ & de \\
\hline & Exportaciones de estados miembros a terceros países & EGS & $\begin{array}{l}\text { Millones } \\
\text { Euros }\end{array}$ & de \\
\hline & Importaciones de estados miembros a terceros países & IGS & $\begin{array}{l}\text { Millones } \\
\text { Euros }\end{array}$ & de \\
\hline & Deuda, Excedente público & GOVDEF & $\%$ GDP & \\
\hline & Producción en la industria & PROIND & $\begin{array}{l}\text { l } n \text { d e } x \\
2010=100 \%\end{array}$ & , \\
\hline & Producción en la construcción & PROCON & $\begin{array}{l}\text { l n d e } x \\
2010=100 \%\end{array}$ & , \\
\hline & Participación en las importaciones por miembro de la UE & SHAIMPT & $\%(E U=100 \%)$ & \\
\hline & Participación en las Exportaciones por miembro de la UE & SHAEXPO & $\%(E U=100 \%)$ & \\
\hline
\end{tabular}




\begin{tabular}{|c|c|c|c|}
\hline Categoría & Indicador & Variable & $\begin{array}{l}\text { Unidad } \\
\text { medida }\end{array}$ \\
\hline \multirow{9}{*}{ 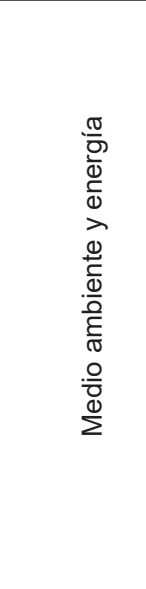 } & Emisiones de SOx procedentes del transporte y comunicación & AETSO & Toneladas \\
\hline & $\begin{array}{l}\text { Emisiones de gases de efecto invernadero procedentes del } \\
\text { transporte }\end{array}$ & GHGTR & $\begin{array}{l}\text { Miles } \\
\text { toneladas }\end{array}$ \\
\hline & Impuestos ambientales sobre transporte & ETXTRANS & $\begin{array}{l}\text { Millones } \\
\text { Euros }\end{array}$ \\
\hline & Generación total de residuos & GENWAS & Toneladas \\
\hline & Precios del gas natural de uso industrial & GPICONS & $\begin{array}{l}\text { Euro / Kilowatt- } \\
\text { hora }\end{array}$ \\
\hline & $\begin{array}{l}\text { Residuos de aparatos eléctricos y electrónicos producidos } \\
\text { por hogares }\end{array}$ & WELEC & $\begin{array}{l}\text { Kilogramos per } \\
\text { cápita }\end{array}$ \\
\hline & Productividad de los recursos & REPRO & Euro / Kilogramo \\
\hline & $\begin{array}{l}\text { Emisiones de gases de efecto invernadero procedentes de } \\
\text { la agricultura }\end{array}$ & GHGAGR & $\begin{array}{l}\text { Millones } \\
\text { toneladas }\end{array}$ \\
\hline & Áreas de control de emisión de azufre & SECA & Número \\
\hline \multirow{6}{*}{ 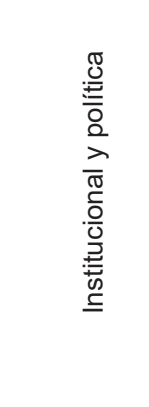 } & Ubicación geográficas de las costas & SFRONT & Número \\
\hline & $\begin{array}{l}\text { Capacidad de las terminales de regasificación de GNL en } \\
\text { operación }\end{array}$ & $\mathrm{RCO}$ & $\begin{array}{l}\text { billones } \mathrm{m}^{3} \text { por } \\
\text { año }\end{array}$ \\
\hline & $\begin{array}{l}\text { Capacidad de las terminales de regasificación de GNL en } \\
\text { construcción }\end{array}$ & $\mathrm{RCU}$ & $\begin{array}{l}\text { billones } \mathrm{m}^{3} \text { por } \\
\text { año }\end{array}$ \\
\hline & $\begin{array}{l}\text { Capacidad de las terminales de regasificación de GNL en } \\
\text { planes }\end{array}$ & $\mathrm{RCP}$ & $\begin{array}{l}\text { billones } \mathrm{m}^{3} \text { por } \\
\text { año }\end{array}$ \\
\hline & Forma de gobierno & FDG & Número \\
\hline & Modelos de gestión portuaria en Europa & EPG & Número \\
\hline \multirow{7}{*}{ 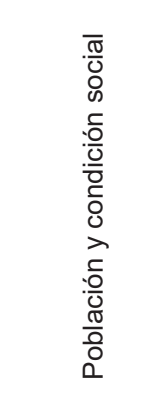 } & Gastos totales en investigación y desarrollo & RDEXPN & Euro / habitante \\
\hline & Población & POBL & Habitantes \\
\hline & Esperanza de vida & LIFEEXP & Edad \\
\hline & Población en riesgo de pobreza o exclusión social & RISKPOV & $\begin{array}{l}\text { Porce n t a j e } \\
\text { población }\end{array}$ \\
\hline & Tasa de desempleo & UNER & $\begin{array}{l}\text { Porcentaje } \\
\text { población activa }\end{array}$ \\
\hline & Estudiantes matriculados en educación terciaria & STENTER & Población \\
\hline & Gastos en protección social & EXPSPBE & Euro / habitante \\
\hline
\end{tabular}

\section{Fase 2: Construcción del modelo}

El siguiente paso, tras haber seleccionado las variables fue la discretización de las mismas, esto permitió la construcción del modelo, dado que las variables discretas o nominales son las más comunes en el caso de redes bayesianas, pues, aunque existen modelos de redes bayesianas con variables continuas, éstos se limitan a variables gaussianas y relaciones lineales.

La discretización consistió en dividir el rango de las variables continuas en un número finito de intervalos exhaustivos y exclusivos. Para discretizar los atributos fue necesario seleccionar el número de intervalos. Los intervalos se dividen teniendo en cuenta la frecuencia, es decir, el número de instancias en cada intervalo o utilizando el mismo rango, es decir, la misma distancia, siempre se pierde información al discretizar. Para evitar esto último. Normalmente 
las redes bayesianas consideran variables discretas o nominales, por lo que si no lo son, hay que discretizarlas antes de construir el modelo. Aunque existen modelos de redes bayesianas con variables continuas, éstos están limitados a variables gaussianas y relaciones lineales.

Un paso clave en la implementación de redes bayesianas es la discretización de variables continuas. Existen varios métodos matemáticos para construir distribuciones discretas, los cuales dependen del software utilizado. Para este estudio se ha considerado variables discretas para lo cual se han discretizado las variables continuas atendiendo al criterio de percentiles, usando los percentiles p25, p50 y p75 que han dado buenos resultados en otros estudios de similares características.

Una vez discretizadas las variables, el aprendizaje estructural se enfatizó en encontrar las relaciones de dependencia entre las variables, de tal forma que se logró determinar la topología de la red bayesiana. Dependiendo del tipo de estructura, es posible aplicar distintos métodos de aprendizaje estructural: redes multiconectadas, aprendizaje de árboles, aprendizaje de poli-árboles, aprendizaje de métodos basados en medidas y búsqueda y métodos basados en relaciones de dependencia.

Aprender automáticamente la estructura gráfica de una red bayesiana es un desafío que se persigue dentro del aprendizaje automático. La red obtenida a partir de las variables seleccionadas, usando el algoritmo K2, es la que se representa en la Figura 4.

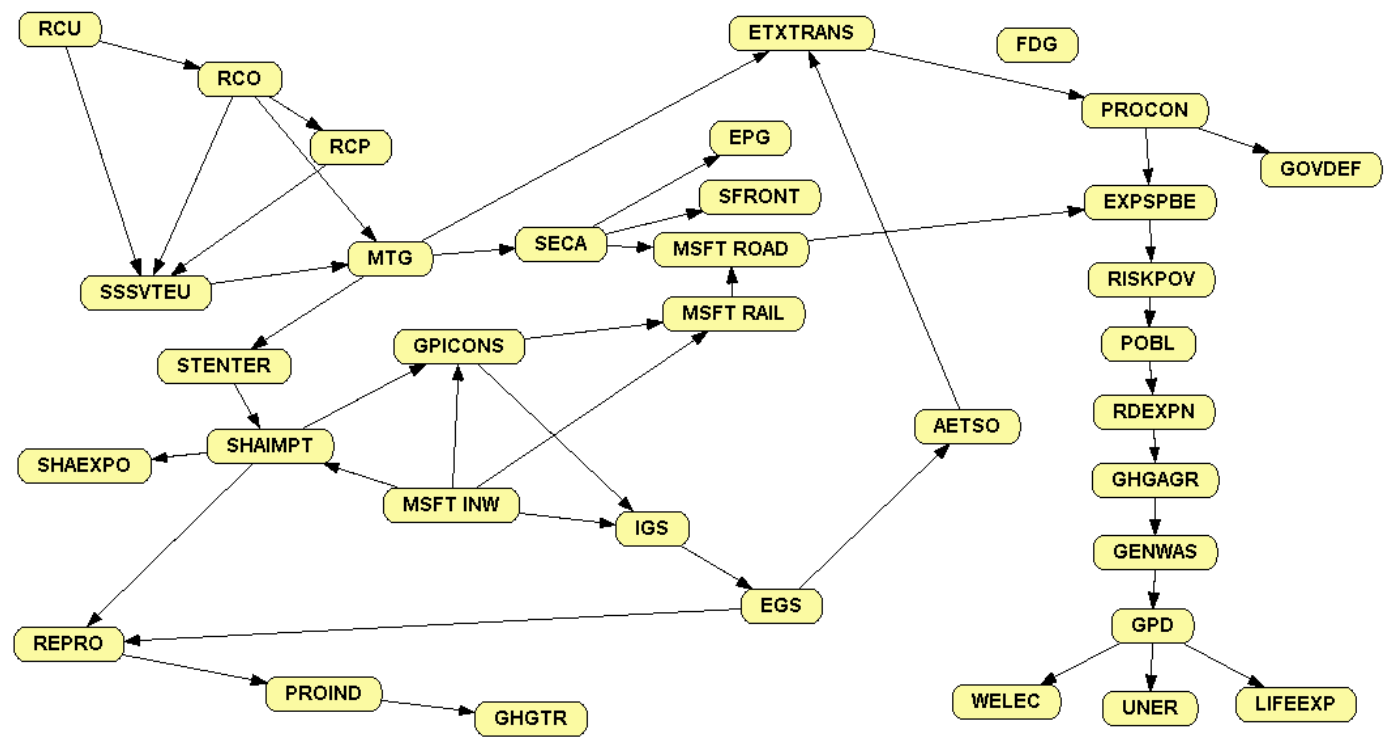

Figura 4. Representación gráfica de la red obtenida a partir de las variables seleccionadas

Para la construcción de la red bayesiana, se empleó el software Elvira, el cual permite representar redes bayesianas y diagramas de Influencia. Existen cuatro apartados básicos en un fichero de especificación de redes Elvira (normalmente con extensión .elv)

1. Descripción de la Red

2. Descripción de los nodos de la red.

3. Descripción de los enlaces entre los nodos.

4. Descripción de las relaciones de la red. 
A partir de una estructura de la red bayesiana inicial, el algoritmo K2 busca en el espacio de la estructura de la red bayesiana generada y selecciona la estructura que maximiza la métrica $\mathrm{K} 2$.

La construcción precisa del clasificador de red bayesiano de la base de datos es un problema NP-difícil y sigue siendo uno de los desafíos más interesantes. El algoritmo K2 puede reducir el espacio de búsqueda de forma efectiva, mejorar la eficiencia de aprendizaje, pero requiere el pedido inicial de nodos como entrada, que está muy limitado por la ausencia de información a priori. Por otro lado, el proceso de búsqueda del algoritmo K2 usa una estrategia de búsqueda codiciosa y las soluciones son fáciles de caer en la optimización local. En este trabajo, presentamos una estructura de red bayesiana mejorada de aprendizaje con ordenamiento de nodos a través del algoritmo K2. Este algoritmo genera un ordenamiento eficaz de nodos como entrada basada en información mutua condicional. El algoritmo K2 también se mejora al combinarse con el algoritmo de recocido simulado para evitar caer en la optimización local. Los resultados experimentales en dos redes de referencia Asia y Alarma muestran que este nuevo algoritmo mejorado tiene una mayor precisión de clasificación y un mejor grado de coincidencia de datos (Wei et al., 2014).

El algoritmo S\&S K2 (Cooper \& Herskovits, 1992) utiliza una búsqueda codiciosa y no puede imponer ninguna restricción sobre el número de padres que tiene un nodo. La búsqueda de K2 comienza asumiendo que un nodo (que representa una variable discreta) no tiene padres y luego agrega incrementalmente ese elemento primario de un orden determinado cuya adición aumenta al máximo el puntaje de la estructura resultante. Dejamos de agregar padres al nodo cuando el puntaje se detiene para aumentar.

Una métrica de puntuación común es la puntuación Bayesiana que es, en principio, la probabilidad posterior de una estructura $G$ dada una muestra aleatoria $D=\left\{d_{1}, d_{2}, \ldots, d_{N}\right\}$ de la distribución conjunta de $\mathrm{X}$

$$
\begin{array}{r}
P(\mathcal{G} \mid D)=\frac{P(D \mid \mathcal{G}) P(\mathcal{G})}{P(D)}=\frac{P(\mathcal{G}, D)}{P(D)} \\
P(\mathcal{G}, D)=P(\mathcal{G}) \prod_{i=1}^{n} \prod_{j=1}^{q_{i}} \frac{\Gamma\left(\alpha_{i j}\right)}{\Gamma\left(\alpha_{i j}+N_{i j}\right)} \prod_{k=1}^{r_{i}} \frac{\Gamma\left(\alpha_{i j k}+N_{i j k}\right)}{\Gamma\left(\alpha_{i j k}\right)}
\end{array}
$$

Suponiendo que los parámetros asociados con cada variable son mutuamente independientes, la métrica $\mathrm{K} 2$ es descomponible. Es decir, la métrica se puede escribir como un producto de calificaciones independientes, $g\left(\mathrm{X}_{\mathrm{i}}, \mathrm{P}_{\mathrm{ai}}\right)$, una para cada variable y su grupo de padres (que mide el grado de dependencia entre la variable y sus padres), en la forma

$$
P(\mathcal{G}, D)=P(\mathcal{G}) \prod_{i=1}^{n} g\left(X_{i}, \mathbf{P a}_{i}\right)
$$

Como un algoritmo de búsqueda heurístico representativo de algoritmos de aprendizaje de estructura para la red bayesiana, el algoritmo K2 se atrapa fácilmente en el óptimo local en el proceso de optimización de la estructura de red bayesiana. 


\section{Fase 3: Interpretación de la red}

La última etapa de la metodología seguida consiste en el análisis e interpretación de la relaciones entre las variables obtenidas tras la construcción del modelo. Así, se estudia la red y se descompone en pequeñas relaciones para su estudio. Las redes de creencia o redes bayesianas contemplan tres tipos de relaciones (Figura 5).

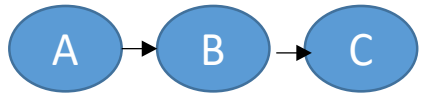

Conexión serial

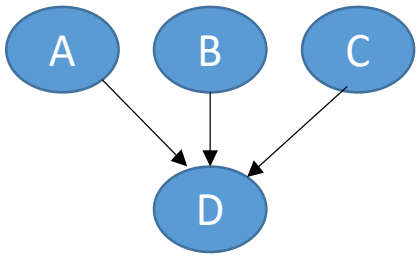

Conexión convergente

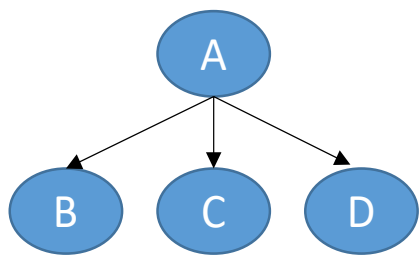

Conexión divergente

Figura 5. Tipos de conexiones en las redes Bayesianas.

Fuente: (Molina et al., 2017)

A partir de dichas conexiones se establecen las siguientes relaciones entre las variables:
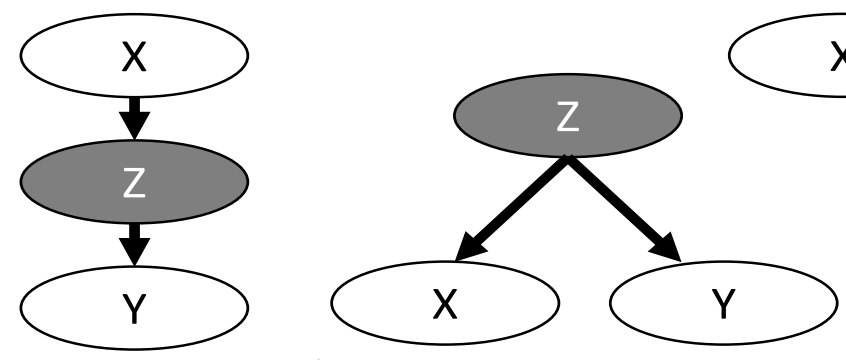

a

b

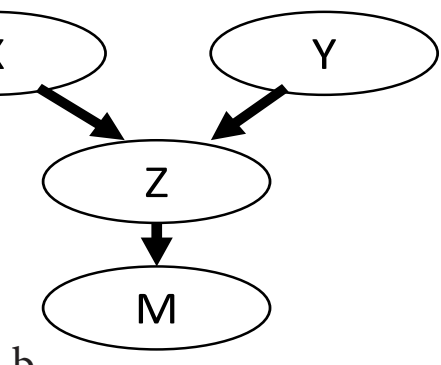

Figura 6. a. d-separación de nodos $X$ e $Y$ b. d-conexión de nodos $X$ e $Y$

- Los nodos $\mathrm{X}$ e $\mathrm{Y}$ están d-separados si cualquier relación (indirecta) entre $\mathrm{X}$ e $\mathrm{Y}$ hay una variable $Z$, tal que (Figura 6.1)

- $\quad$ Z está en conexión serial o divergente y es conocida, o

- $\quad$ Z está en conexión convergente y ni Z o alguno de sus hijos son conocidos

- Los nodos $\mathrm{X}$ e $\mathrm{Y}$ están d-conectados si no están separados (existe una relación indirecta entre $\mathrm{X}$ e $\mathrm{Y}$ y no están d-separados por ningún otro nodo o conjunto de nodos) (Figura 6.2)

- $\quad$ Si los nodos $X$ e $Y$ están d-separados por $Z$, entonces $X$ e $Y$ son condicionalmente independientes, dado Z (Pearl, 1988)

Es en esta etapa también cuando se identifican aquellas que, por estar descolgadas de la 
red, no guardan ninguna relación con el resto de variables consideradas.

\section{RESULTADOS Y DISCUSIÓN}

A partir de los datos representados en la Figura 4 es posible inferir que la variable FDG (Forma de gobierno) no posee relación alguna con las variables que conforman la red y por lo tanto no es ni causa ni efecto de ninguna de ellas. La variable forma de gobierno hace referencia a los cuatro tipos de formas de gobiernos existente en la Unión Europea:

- $\quad$ República Presidencialista

- $\quad$ República Semipresidencialista

- $\quad$ República Parlamentaria

- Monarquía Parlamentaria

Se pretendía con la elección de esta variable determinar en el estudio la influencia de la forma de gobierno en el panorama del transporte asociado al transporte marítimo de corta distancia y al empelo de combustibles como GNL, pero la red resultante no ha encontrado relación con esta variable y el resto. En este caso el nodo representa una variable que es independiente, ya que no tiene arcos con ninguna otra. Se llama a esto independencia total.

Asimismo, es posible identificar que RCU (Capacidad de las terminales de regasificación de GNL en construcción) y MSFT INW (Distribución modal de transporte de carga por aguas continentales) son los dos nodos raíz de la red, al ser las únicas que son padres y no hijos de otras variables; la primera es del tipo comercio internacional y transporte y la segunda del tipo institucional y político (Tabla 2).

Tabla 2: Padres de la red

\begin{tabular}{|l|c|}
\hline \multirow{2}{*}{ Padres de la red } & $\begin{array}{c}\text { RCU (Capacidad de las terminales de regasificación de } \\
\text { GNL en construcción) }\end{array}$ \\
\cline { 2 - 3 } & $\begin{array}{c}\text { MSFT INW (Distribución modal de transporte de carga } \\
\text { por aguas continentales }\end{array}$ \\
\hline
\end{tabular}

Por ello respecto al estudio de GNL para el transporte marítimo de corta distancia en Europa y en base a la red construida las variables fundamentales para el planificador son los padres de la red, ya que el resto depende de ellas en mayor o menor medida. Por lo que a priori la capacidad de las terminales de regasificación de GNL en construcción y la distribución modal de transporte de carga por aguas continentales son las variables más determinantes en la elección del GNL para transporte marítimo de corta distancia en Europa.

Tanto la variable RCU como la MSFT INW son variables decisoras, cada una de ellas aparecen en la red como una variable "nodo" de la que salen arcos, por lo que se genera en cada una de ellas conexiones divergentes. En el caso de la primera se tiene un nodo padre que proyecta todos sus arcos hacia 2 hijos. Esto quiere decir que RCO (Capacidad de las terminales de regasificación de GNL en operación) y SSSVTEU (TEU's en Short Sea Shipping) son consecuencia de RCU (Capacidad de las terminales de regasificación de GNL 
en construcción) como se aprecia en la Figura 4. Así, el planificador ha de considerar las terminales regasificadoras en construcción ejercerán influencia sobre la capacidad actual y sobre los TEUS que actualmente se transportan por transporte marítimo de corta distancia de forma directa, según las relaciones obtenidas de la construcción de la red.

Lo mismo sucede con la variable MSFT INW (Distribución modal de transporte de carga por aguas continentales) en la cual, las flechas o arcos salen de él y divergen hacia sus 4 hijos, por lo que IGS (Importaciones de estados miembros a terceros países), GPICONS (Precios del gas natural de uso industrial), MSFT RAIL (Distribución modal de transporte de carga por ferrocarril) y SHAIMPT (Participación en las importaciones por miembro de la UE) son consecuencia de MSFT INV. Por lo que si el planificador conoce la distribución modal de transporte de carga por aguas continentales o pretende ver qué pasaría si modifica el reparto modal, con las relaciones obtenidas con las red bayesiana dispone de información directa sobre las importaciones de estados miembros a terceros países, sobre los precios del gas natural de uso industrial así como de la distribución modal de transporte de carga por ferrocarril de la participación en las importaciones por miembro de la UE al darse una relación divergente En las conexiones divergentes, también conocidas como clasificadores ingenuos de Bayes existe un nodo padre (o clase) que proyecta sus arcos sobre varios hijos. Este tipo de conexión es el más apropiado para representar procesos de diagnóstico y son útiles para estimar el conocimiento asociado a conceptos en función del comportamiento del usuario. Cuando no conocemos el estado de la variable madre existe dependencia entre las variables hijas. Sin embargo, cuando el estado de esta variable se conoce, las evidencias sobre las variables hijas no se propagarán entre ellas. En nuestro el caso que se estudiar se puede decir que las importaciones de estados miembros a terceros países, los precios del gas natural de uso industrial, la distribución modal de transporte de carga por ferrocarril y la participación en las importaciones por miembro de la UE son independientes si se conoce la distribución modal de transporte de carga por aguas continentales.

Se van a estudiar las relaciones que se producen entre las variables económicas como se destacan en la Figura 7.

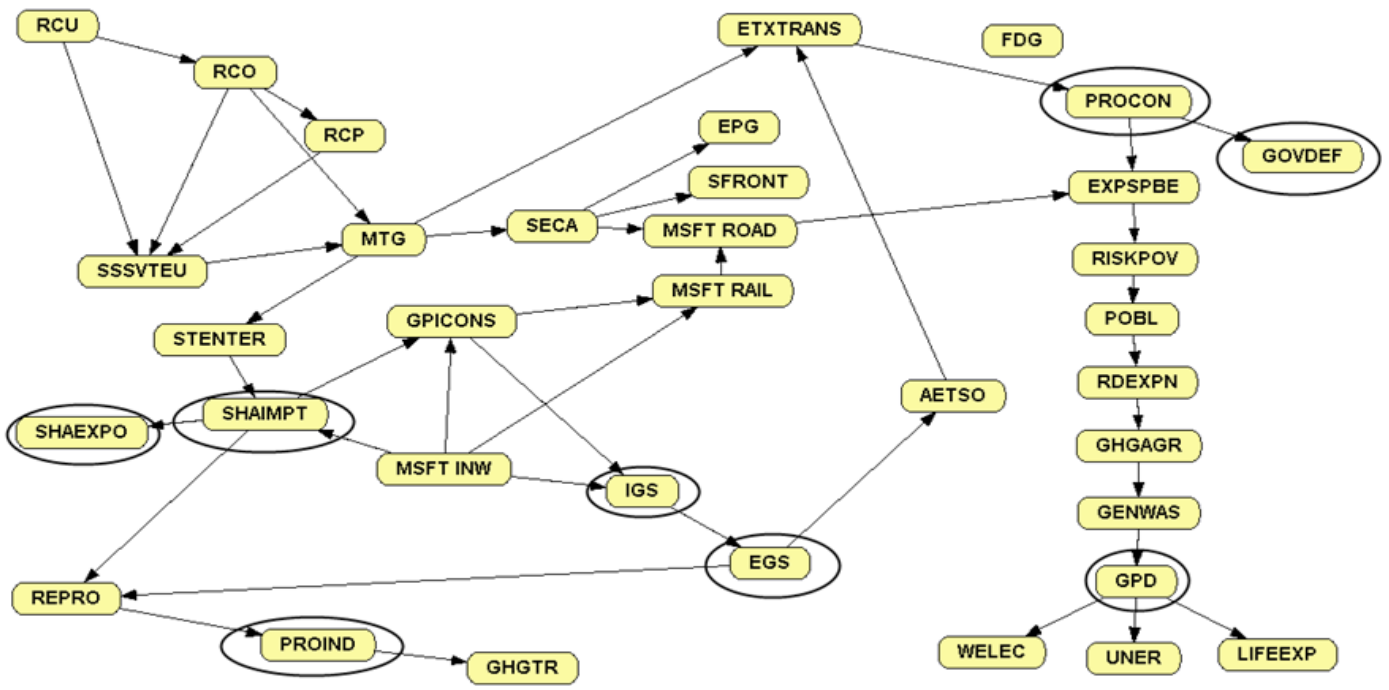

Figura 7. Representación gráfica de la red obtenida. Indicación de las variables económicas utilizadas en el estudio 
Al analizar la siguiente relación entre variables se observa no solamente relaciones divergentes sino también convergentes en torno a la variable SHAIMPT (Participación en las importaciones por miembro de la UE). Esta variable tiene dos padres, es decir, las variables STENTER (Estudiantes matriculados en educación terciaria) y MSFT INW (Distribución modal de transporte de carga por aguas continentales) convergen en SHAIMPT, en otras palabras son dos causas alternativas de SHAIMPT (Tabla 3). En este tipo de conexiones las variables padre son independientes entre sí, sin embargo si se tiene la evidencia sobre la variable hijo, las variables padre se tornaran dependientes (Figura 8). Así se puede decir que de la red resultante la participación en las importaciones por miembro de la UE tienen su causa en los estudiantes matriculados en educación terciaria) y en la distribución modal de transporte de carga por aguas continentales

Tabla 3. Padres e hijos de la variable SHAIMPT (Participación en las importaciones por miembro de la UE)

\begin{tabular}{|c|c|}
\hline $\begin{array}{c}\text { Padres de SHAIMPT (Participación en las importaciones } \\
\text { por miembro de la UE) }\end{array}$ & $\begin{array}{c}\text { Hijos de SHAIMPT (Participación en las importaciones } \\
\text { por miembro de la UE) }\end{array}$ \\
\hline $\begin{array}{c}\text { STENTER (Estudiantes matriculados en educación } \\
\text { terciaria) } \\
\text { Categoría: población y condición social }\end{array}$ & $\begin{array}{c}\text { GPICONS (Precios del gas natural de uso industrial) } \\
\text { Categoría: medio ambiente y energía }\end{array}$ \\
\hline $\begin{array}{c}\text { MSFT INW (Distribución modal de transporte de carga } \\
\text { por aguas continentales) }\end{array}$ & $\begin{array}{c}\text { SHAEXPO (Participación en las Exportaciones por } \\
\text { miembro de la UE) }\end{array}$ \\
Categoría: comercio internacional y transporte & $\begin{array}{c}\text { Categonas economía y finanzas } \\
\text { REPRO (Productividad de los recursos) } \\
\text { Categoría: medio ambiente y energía }\end{array}$ \\
\hline
\end{tabular}

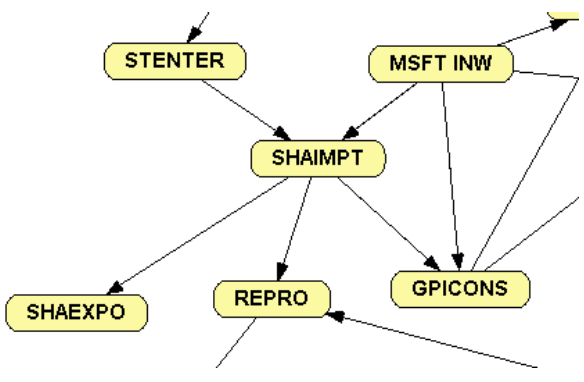

Figura 8. Relaciones establecidas con la variable SHAIMPT (Participación en las importaciones por miembro de la UE)

Con respecto a las relaciones divergentes, la variable SHAIMPT es una variable decisoria, pues aparece en la red como una variable "nodo" de la que salen 3 arcos que divergen hacia sus hijos: SHAEXPO (Participación en las Exportaciones por miembro de la UE), REPRO (Productividad de los recursos) y GPICONS (Precios del gas natural de uso industrial), es 
decir estas 3 variables son un efecto indirecto de STENDER y MSFT INW. Así, en términos de causa y efecto, la participación en las importaciones por miembro de la UE inciden de forma directa (son causa) de la participación en las exportaciones por miembro de la UE, de la productividad de los recursos y de los precios del gas natural de uso industrial, siendo variables de categoría económica y medioambiental.

Por tanto, la variable económica SHAIMPT (Participación en las importaciones por miembro de la UE) depende de MSFT INW (Distribución modal de transporte de carga por aguas continentales) y del nivel educativo del país a través de la variable de STENDER (Estudiantes matriculados en educación terciaria). Si se estudia desde un punto de vista de redes causales se diría que SHAIMPT es consecuencia de MSFT INW y de STENDER. Por otro lado SHAEXPO (Participación en las Exportaciones por miembro de la UE), REPRO (Productividad de los recursos) y GPICONS (Precios del gas natural de uso industrial) son consecuencia de SHAIMPT así como de MSFT INW y de STENDER a través de SHAIMPT (Tabla 4).

Tabla 4. Relaciones en serie entre variables. Caso 1

\begin{tabular}{|c|c|c|}
\hline A & B & C \\
\hline $\begin{array}{l}\text { STENTER (Estudiantes matriculados } \\
\quad \text { en educación terciaria) } \\
\text { Categoría: población y condición social }\end{array}$ & $\begin{array}{l}\text { SHAIMPT (Participación en las } \\
\text { importaciones por miembro de la UE) }\end{array}$ & $\begin{array}{c}\text { GPICONS (Precios del gas } \\
\text { natural de uso industrial) } \\
\text { Categoría: medio ambiente y } \\
\text { energía }\end{array}$ \\
\hline $\begin{array}{l}\text { STENTER (Estudiantes matriculados } \\
\text { en educación terciaria) } \\
\text { Categoría: población y condición social }\end{array}$ & $\begin{array}{l}\text { SHAIMPT (Participación en las } \\
\text { importaciones por miembro de la UE) }\end{array}$ & $\begin{array}{c}\text { SHAEXPO (Participación en } \\
\text { las Exportaciones por miembro } \\
\text { de la UE) } \\
\text { Categoría: economía y } \\
\text { finanzas }\end{array}$ \\
\hline $\begin{array}{l}\text { STENTER (Estudiantes matriculados } \\
\text { en educación terciaria) } \\
\text { Categoría: población y condición social }\end{array}$ & $\begin{array}{l}\text { SHAIMPT (Participación en las } \\
\text { importaciones por miembro de la UE) }\end{array}$ & $\begin{array}{l}\text { REPRO (Productividad de los } \\
\text { recursos) } \\
\text { Categoría: medio ambiente y } \\
\text { energía }\end{array}$ \\
\hline $\begin{array}{c}\text { MSFT INW (Distribución modal } \\
\text { de transporte de carga por aguas } \\
\text { continentales) } \\
\text { Categoría: comercio internacional y } \\
\text { transporte }\end{array}$ & $\begin{array}{l}\text { SHAIMPT (Participación en las } \\
\text { importaciones por miembro de la UE) }\end{array}$ & $\begin{array}{c}\text { GPICONS (Precios del gas } \\
\text { natural de uso industrial) } \\
\text { Categoría: medio ambiente y } \\
\text { energía }\end{array}$ \\
\hline $\begin{array}{l}\text { MSFT INW (Distribución modal } \\
\text { de transporte de carga por aguas } \\
\text { continentales) } \\
\text { Categoría: comercio internacional y } \\
\text { transporte }\end{array}$ & $\begin{array}{l}\text { SHAIMPT (Participación en las } \\
\text { importaciones por miembro de la UE) }\end{array}$ & $\begin{array}{c}\text { SHAEXPO (Participación en } \\
\text { las Exportaciones por miembro } \\
\text { de la UE) } \\
\text { Categoría: economía y } \\
\text { finanzas }\end{array}$ \\
\hline $\begin{array}{c}\text { MSFT INW (Distribución modal } \\
\text { de transporte de carga por aguas } \\
\text { continentales) } \\
\text { Categoría: comercio internacional y } \\
\text { transporte }\end{array}$ & $\begin{array}{l}\text { SHAIMPT (Participación en las } \\
\text { importaciones por miembro de la UE) }\end{array}$ & $\begin{array}{l}\text { REPRO (Productividad de los } \\
\text { recursos) } \\
\text { Categoría: medio ambiente y } \\
\text { energía }\end{array}$ \\
\hline
\end{tabular}

De la misma forma RISKPOV (Población en riesgo de pobreza o exclusión social) depende de EXPSPBE (Gastos en protección social) y POBL (Población) depende de RISKPOV y así sucesivamente hasta llegar al final de la serie. Por otro lado, en la Figura 9, se observa otra conexión serial más corta, en donde la variable REPRO (Productividad de los recursos) tiene 2 padres de los cuales depende: SHAIMPT (Participación en las importaciones por miembro de la UE) y EGS (Exportaciones de estados miembros a terceros países), y tiene un hijo PROIND (Producción en la industria) quien depende de REPRO y finalmente GHGTR (Emisiones de gases de efecto invernadero procedentes del transporte) quien depende 
de PROIND (Tabla 5). Así las Emisiones de gases de efecto invernadero procedentes del transporte en términos causales y por la red obtenida se ven afectados de forma directa por la producción en la industria y de forma indirecta a través de producción en la industria por la Productividad de los recursos.

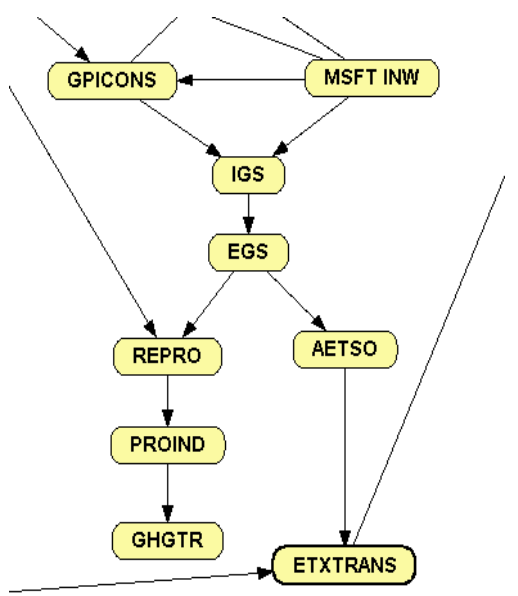

Figura 9. Relaciones establecidas con la variable EGS (Exportaciones de estados miembros a terceros países)

Tabla 5. Relaciones en serie entre variables. Caso 2

\begin{tabular}{|c|c|c|}
\hline A & B & C \\
\hline $\begin{array}{l}\text { REPRO (Productividad de los } \\
\text { recursos) }\end{array}$ & $\begin{array}{l}\text { PROIND (Producción en la } \\
\text { industria) }\end{array}$ & $\begin{array}{c}\text { GHGTR (Emisiones de } \\
\text { gases de efecto invernadero } \\
\text { procedentes del transporte) }\end{array}$ \\
\hline $\begin{array}{l}\text { REPRO (Productividad de los } \\
\text { recursos) }\end{array}$ & $\begin{array}{l}\text { PROIND (Producción en la } \\
\text { industria) }\end{array}$ & GOVDEF \\
\hline $\begin{array}{l}\text { IGS (Importaciones de estados } \\
\text { miembros a terceros países) }\end{array}$ & $\begin{array}{l}\text { EGS (Exportaciones de estados } \\
\text { miembros a terceros países) }\end{array}$ & $\begin{array}{l}\text { AETSO (Emisiones de SOx } \\
\text { procedentes del transporte y } \\
\text { comunicación) }\end{array}$ \\
\hline
\end{tabular}

Entre las variables GPICONS (Precios del gas natural de uso industrial) MSFTINW (Distribución modal de transporte de carga por aguas continentales) y la variable IGS (Importaciones de estados miembros a terceros países) se produce una relación convergente las variables madre son independientes entre sí, pero una variable de convergencia puede influir sobre sus nodos padre. La propiedad importante de este tipo de conexión a la hora de propagar la información es que cuando se tiene evidencia sobre la variable de convergencia, los nodos padre se vuelven dependientes y la evidencia del estado de uno de ellos se propaga por los demás. Así en el caso de estudio los precios del gas natural de uso industrial y la Distribución modal de transporte de carga por aguas continentales son dependientes entre ellos si se conocen las importaciones de estados miembros a terceros países

Por otra parte, en la red se observa una serie de relaciones entre variables que se denominan conexiones seriales. En la Figura 10, se aprecia que la variable EXPSPBE (Gastos en protección social) depende de MSFT ROAD (Distribución modal de transporte de carga 
por carretera) y de PROCON (Producción en la construcción) y esta última depende de ETXTRANS (Impuestos ambientales sobre transporte) y así sucesivamente. En término de causa efecto, tal y como se muestra en la Tabla 6, se diría que ETEXTRANS es causa de PROCON y que PROCON es causa de EXPSPBE. En este caso, dada la dependencia entre las variables, cuando conocemos información sobre ETXTRANS podemos modificar nuestra certeza sobre el estado de EXPSPBE; y a la inversa, cuando sabemos algo sobre el estado de EXPSPBE la creencia sobre el estado de ETXTRANS se altera.

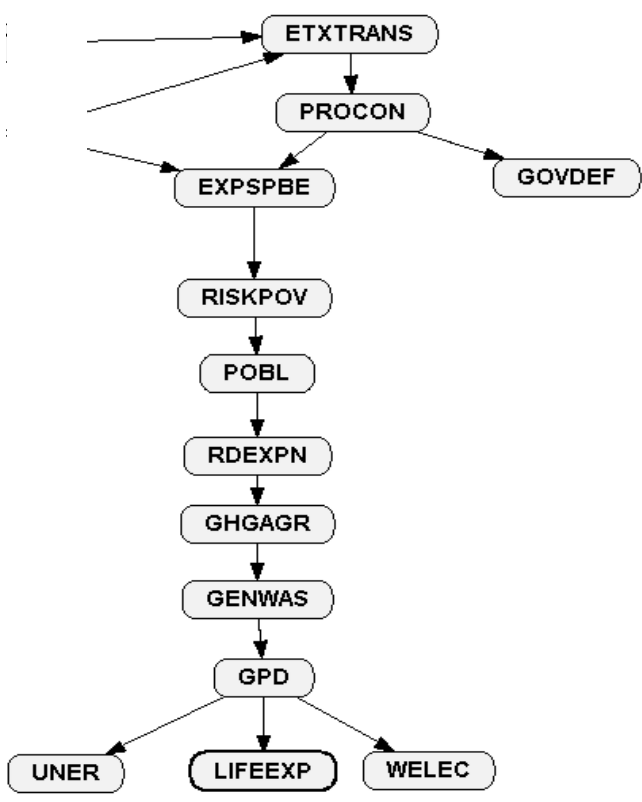

Figura 10. Relaciones establecidas con la variable EXPSPBE (Gastos en protección social)

Tabla 6. Relaciones en serie entre variables. Caso 3

\begin{tabular}{|c|c|c|}
\hline A & B & C \\
\hline $\begin{array}{c}\text { ETXTRANS (Impuestos } \\
\text { ambientales sobre transporte) }\end{array}$ & $\begin{array}{c}\text { PROCON (Producción en la } \\
\text { construcción) }\end{array}$ & $\begin{array}{c}\text { EXPSPBE (Gastos en } \\
\text { protección social) }\end{array}$ \\
\hline $\begin{array}{c}\text { ETXTRANS (Impuestos } \\
\text { ambientales sobre transporte) }\end{array}$ & $\begin{array}{c}\text { PROCON (Producción en la } \\
\text { construcción) }\end{array}$ & $\begin{array}{c}\text { PROIND (Producción en la } \\
\text { industria) }\end{array}$ \\
\hline $\begin{array}{c}\text { GENWAS (Generación total de } \\
\text { residuos) }\end{array}$ & GDP (Producto interno bruto) & UNER (Tasa de desempleo) \\
\hline $\begin{array}{c}\text { GENWAS (Generación total de } \\
\text { residuos) }\end{array}$ & GDP (Producto interno bruto) & LIFEEXP (Esperanza de vida) \\
\hline $\begin{array}{c}\text { GENWAS (Generación total de } \\
\text { residuos) }\end{array}$ & GDP (Producto interno bruto) & $\begin{array}{c}\text { WELEC (Residuos de aparatos } \\
\text { eléctricos y electrónicos } \\
\text { producidos por hogares) }\end{array}$ \\
\hline
\end{tabular}

Entre la variable GDP (Producto interno bruto) se produce una conexión divergente con las variables UNER (Tasa de desempleo), LIFEEXP (Esperanza de vida), WELEC (Residuos de 
aparatos eléctricos y electrónicos producidos por hogares), en las conexiones divergentes tenemos un nodo padre que proyecta sus arcos hacia varios hijos; o lo que es lo mismo, las flechas que salen de él y divergen hacia sus hijos, cuando no conocemos información sobre el producto interno bruto existe una dependencia entre las variables análoga a la que se da en la conexión serial entre la tasa de desempleo, la esperanza de vida y los residuos de aparatos eléctricos y electrónicos producidos por hogares. Sin embargo, si se conoce el producto interno bruto, cosa bastante probable las variables entre la tasa de desempleo, la esperanza de vida y los residuos de aparatos eléctricos y electrónicos producidos por hogares son independientes.

En vista de los resultado, estableciendo una jerarquía entre las variables, y a partir de los padres de la red, es posible encontrar que la categoría de transporte y comercio internacional y la categoría economía y finanzas son los más importantes, puesto que sus indicadores son los más cercanos a los nodos raíz de la red y, por tanto, son los menos dependientes. Por tanto, se deprende que ambas categorías resultan significativas a la hora de decidir la implementación del GNL como combustible en el tramo marítimo de las autopistas del mar. Por el contrario, las categorías de medio ambiente y energía y de población y condición social son las más dependientes, estando más alejadas de los nodos raíz de la red. (Figura 11)

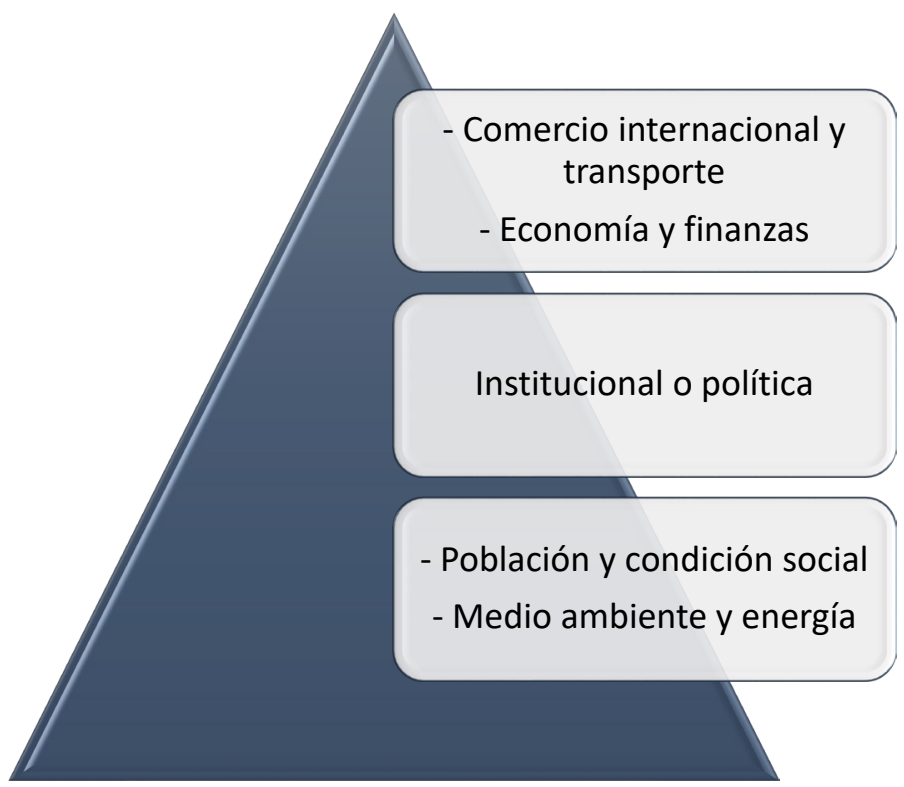

Figura 11. Grado de importancia de las categorías definidas en la elección del gas natural licuado como combustible alternativo en el transporte marítimo de corta distancia en Europa

\section{CONCLUSIONES}

Del estudio de las relaciones entre variables asociadas al transporte marítimo de corta distancia en Europa y el uso de GNL como combustible se desprende que la construcción de un modelo a través de redes de decisión permite establecer las relaciones entre dichas variables en los cinco grupos de trabajo: Comercio internacional y Transporte, Economía y 
Finanzas, Medio Ambiente y Energía, Institucional y Político, Condición Social y Poblacional. Se observa que la categoría de transporte y comercio internacional y la categoría economía y finanzas son los más importantes a la hora de decidir la implementación del GNL como combustible en el short sea shipping.

A partir de la red construida y las relaciones entre las variables que se obtienen, parece que el nivel educativo de la población es un factor determinante, tanto en la participación de las exportaciones como en las importaciones por miembro de la UE, como en la distribución modal del transporte. Respecto a las importaciones y exportaciones de estados miembros a terceros países, se observa que dependen de los precios del gas natural de uso industrial. Se aprecia igualmente que en cualquiera de sus variables, son las importaciones las que tienen efecto sobre las exportaciones a través de otras variables.

Al analizar la variable económica producción en la industria se obtiene que depende de las importaciones y exportaciones de estados miembros a terceros países a través de la productividad de los recursos, medidos en euros por kilogramo.

Al estudiar las relaciones entre las diversas variables de la red y la tipología de conexión que entre ellas de producen se ha observado en base a la red construida que, la producción en la construcción tiene dependencia de los impuestos ambientales sobre transporte, así como la deuda. Asimismo, el excedente público depende de los impuestos ambientales sobre transporte a través de la producción en la construcción. Por tanto, parece que la construcción afecta a la deuda del país y que los impuestos sobre el transporte inciden igualmente sobre ella.

Finalmente, señalar que la variable producto interno bruto está muy al final en la jerarquía de la red, lo que indica que depende de muchas de las variables de las cinco categorías estudiadas. Por ello, en última instancia incide de forma directa sobre tasa de desempleo, la esperanza de vida y los residuos tecnológicos.

\section{REFERENCIAS}

ACEDO, A.; ALMAZÁN, J. Pasado, presente y futuro de las Autopistas del Mar en Europa. Revista de Obras Públicas, 2015, 3565, 31- 38.

ALMAZÁN, J. L.; PALOMINO, M. C.; GONZÁLEZ, N.; SOLER, F. Y IRIBARREN, E. Estimation of Spanish bunkering at EU level of SECAS. Research Conference In Technical Disciplines, Section Transport and Logistic, 2013, 136-141.

BRYNOLF, S.; ANDERSSON K. E. Y FRIDELL, E. A comparative life cycle assessment of marine fuels: liquefied natural gas and three other fossil fuels. Proceedings of the Institution of Mechanical Engineers, Part M: Journal of Engineering for the Maritime Environment, 2011, 225(2), 97-110.

CÁNOVAS, B. Short Sea Shipping, una estrategia Europea. Documento de Opinión, No. 70. Instituto Español de Estudios Estratégicos. 2015.

CASTILLO, E., GUTIÉRREZ, J.M. and HADI, A.S. Expert Systems and Probabilistic Network Models. Springer Verlag. 1997 
COMISIÓN EUROPEA. Libro Blanco, la política europea de transportes de cara al 2010: la hora de la verdad (pp. 11- 53). COM (2001) 370 final. Bruselas: Commission of the European Communities.2001

COMISIÓN EUROPEA. Libro Verde, estrategia europea para una energía sostenible, competitiva y segura (pp. 3- 19). COM (2006) 105 final. Bruselas: Commission of the European Communities. 2006

COOPER, G. F. and HERSKOVITS, E. A Bayesian method for the induction of probabilistic networks from data. Machine learning, 1992, 9(4), 309-347.

CORBETT, J. J.; FISCHBECK, P. S. and PANDIS, S. N. Global Nitrogen and Sulfur Inventories for Oceangoing Ships. Journal of Geophysical Research, 1999, 104(D3), 3457-3470.

CORBETT, J. J. and KORHLER, H. W. Updated Emissions from Ocean Shipping. Journal of Geophysical Research, 2003, 108(D20), pp. 4650.

CORBETT, J. J.; WANG, H. and WINEBRAKE, J. J. The Effectiveness and Costs of Speed Reductions on Emissions from International Shipping. Transportation Research Part D: Transport and Environment, 2009, 14(8), 593-598.

DEVANNEY, J. CO2 Emissions from Shipping: The Case for Taking our Time. 2010. Disponible en: http://www.c4tx.org/ctx/pub/take_time.Pdf

DEVANNEY, J. Y BEACH, S. Detailed Studies of the Impact of Eedi on VIcc Design and co2 Emissions. Center for Tankship Excellence. 2010. Disponible en: http://www.c4tx.org/ctx/pub/ eedi_vlcc.pdf

DEVANNEY, J. Y BEACH, S. Efficient, Safe Reduction of co2 Emissions from Shipping. Center for Tankship Excellence. 2010. Disponible en: http://www.c4tx.org/ctx/pub/tax_vs_ets.pdf

ENDRESEN, Ø., et al.. The Environmental Impacts of Increased International Maritime shipping, Past Trends and Future Perspectives. Global Forum on Transport and Environment in a Globalising World, 2010, Guadalajara, Mexico.

FLORES, F. S., CANCELAS, N. G., ORIVE, A. C., GÁRATE, J. L. A., \& MONZÓN, M. D. C. P. Diseño de un modelo de planificación de zonas de actividades logísticas mediante el empleo de redes bayesianas. Revista ingeniería industrial, 2013, 12(1), 7-26.

GRITSENKO, D. Y YLISKYLÄ-PEURALAHTI, J. Governing shipping externalities: Baltic ports in the process of SOx emission reduction. Maritime Studies, 2013, 12(1),10

GULLBERG, M. Y GAHNSTRÖM, J. North European LNG Infrastructure Project: A feasibility study for an LNG filling station infrastructure and test of recommendations. Draft Feasibility Report. Trans-European Transport Network, 2011

HECKERMAN, D. A tutorial on learning with Bayesian networks. Innovations in Bayesian networks, 2008, pp. 33-82. Springer Berlin Heidelberg.

LASKEY, K. B. Sensitivity analysis for probability assessments in Bayesian networks. In Proceedings of the Ninth Conference on Uncertainty in Artificial Intelligence, 2014 
LIZAZO TORRES, D.; DELFOR MEYER, R. AND TORRES CÁRDENAS, V. Minería De Datos En La Encuesta Permanente De Hogares 2009, Universidad Nacional Del Litoral, Argentina. Revista Ingeniería Industrial, 2011, 10(1), 19-28.

LLOYD, M. Regional action for logistical integration of shipping across Europe (realise). Work package 4. Task 4.1 multi-modal pricing and costing analyses inception report, 2003

MOLINA SERRANO, B., GONZÁLEZ-CANCELAS, N, SOLER-FLORES, F. Y CAMARERO ORIVE, A. Estudio de las relaciones entre variables de transporte y comercio internacional y variables incidentes en la elección del uso del gas natural licuado para Short Sea Shipping mediante un análisis con redes de creencia. Ingeniería Civil, ISSN: 0213-8468, 2017, 188, 57-66

PEARL, J. The Solution for the Branching Factor of the Alpha-Beta Pruning Algorithm and its Optimality. Communications of the ACM, 1982, 25(8), 559-564.

PEARL, J. Probabilistic reasoning in intelligent systems: Networks of plausible reasoning, 1988.

PEARL, J. Bayesian networks. Department of Statistics, UCLA, 2011

PSARAFTIS, H. N. Y KONTOVAS, C. A. Balancing the Economic and Environmental Performance of Maritime Transportation. Transportation Research Part D: Transport and Environment, 2010, 15(8), 458-462.

PUGA, J. L., GARCÍA, J. G., DE LA FUENTE SÁNCHEZ, L., \& DE LA FUENTE SOLANA, E. I. Las redes bayesianas como herramientas de modelado en psicología. Anales de psicología, 2007, 23(2), 307-316.

REALISE. Regional Action for Logistical Integration of Shipping across Europe. The Alliance of Maritime Regional Interests in Europe (AMRIE), GTC2-2000-33032, 2002. Disponible en: http://www.realise-sss.org

REALISE. The Alliance of Maritime Regional Interests in Europe, (AMRIE), Final Report No. GTC2-2000-33032, 2005. Disponible en: http://www.realise-sss.org

RECORDIT. Actions to Promote Intermodal Transport: Final Report No. WP9. 2003. Disponible en: http://www.recordit.org/

RODRÍGUEZ, D., \& DOLADO, J. Redes Bayesianas en la Ingeniería del Software.2007

SHACHTER, R.D. Probabilistic Inference and Influence Diagrams. Probabilistic inference and influence diagrams. Operations Research, 1988, 36(4), 589-604

VASSALLO, W., ET AL. Regional action for logistical integration of shipping across Europe (Realise). 2004.Disponible en: http://www.realise-sss.org/

VERBEEK, R.; KADIJK, G.; MENSCH, V. P.; WULFFERS, C.; BEEMT, B. V. Y FRAGA, F. Environmental and Economic aspects of using $L N G$ as a fuel for shipping in the Netherlands. TNO report. 2011 
WEI Z., XU H., LI W., GUI X., WU X. Improved Bayesian Network Structure Learning with Node Ordering via K2 Algorithm. Huang DS., Jo KH., Wang L. (eds) Intelligent Computing Methodologies. ICIC 2014. Lecture Notes in Computer Science, 2014, 8589. Springer, Cham

\section{BIOGRAFÍA AUTORES}

\section{Beatriz Molina Serrano}

Ingeniero de Caminos, Canales y Puertos por la Universidad Politécnica de Madrid.

Doctorando de la Escuela Técnica Superior de Ingenieros de Caminos, Canales y Puertos de la Universidad Politécnica de Madrid. Es especialista en transporte, planificación y gestión portuaria, redes bayesianas y seguridad vial.

\section{Nicoletta González-Cancelas}

Doctor Ingeniero de Caminos, Canales y Puertos por la Universidad Politécnica de Madrid.

Profesor Contratado Doctor del Departamento de Ingeniería Civil, Transporte y Territorio de la Escuela Técnica Superior de Ingenieros de Caminos, Canales y Puertos de la Universidad Politécnica de Madrid. Es especialista en Logística y transporte. Es profesora del Máster Universitario en Sistemas de Ingeniería Civil (MUSIC) de la Escuela Técnica Superior de Ingenieros de Caminos, Canales y Puertos de la Universidad Politécnica de Madrid y del Master en Gestión y Planificación Portuaria e Intermodalidad; Master del Campus Portuario de Puertos del Estado. Es especialista en Logísitica y Transporte. Pertenece al Grupo de Investigación reconocido de la Universidad Politécnica de Madrid, Grupo de Investigación Logística y Explotación del Transporte y Gestión Operativa. Let \& Go, es responsable de la líneas de investigación en Logística y Data Mining aplicado al transporte.

\section{Francisco Soler-Flores}

Licenciado en Matemáticas por la Universidad de Almería. Doctor en Tecnología y Sistemas de Información por la Universidad de Castilla-La Mancha.

Es Artificial Intelligence Manager en Everis Europa y Profesor en la Facultad de Educación en la Universidad Internacional de La Rioja. Es especialista en Analytics y Machine Learning. 